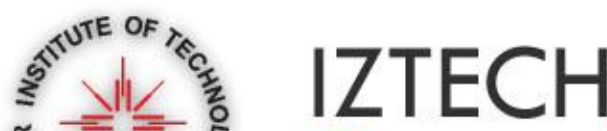

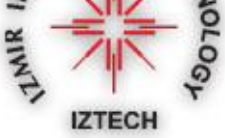 \\ Open Access Articles
}

\section{Controlled Formation of the Two-Dimensional TTBC J-Aggregates in an Aqueous Solution}

The IZTECH Faculty has made this article openly available. Please share how this access benefits you. Your story matters.

\begin{tabular}{|l|l|}
\hline Citation & $\begin{array}{l}\text { Birkan, B, Gülen, D, and Özçelik, S, "Controlled Formation of } \\
\text { the Two-Dimensional TTBC J-Aggregates in an Aqueous } \\
\text { Solution" The Journal of Physical Chemistry B @ } 2006 \\
\text { American Chemical Society }\end{array}$ \\
\hline As Published & $10.1021 /$ jp0573846 \\
\hline Publisher & American Chemical Society \\
\hline Version & PUBLISHED ARTICLE \\
\hline Accessed & FRI JULY 5 16:55:55 GMT 2013 \\
\hline Citable Link & http://hdl.handle.net/11147/ \\
\hline Terms of Use & $\begin{array}{l}\text { Article is made available in accordance with the publisher's } \\
\text { policy and may be subject to Turkish copyright law. Please } \\
\text { refer to the publisher's site for terms of use. }\end{array}$ \\
\hline Detailed Terms & \\
\hline
\end{tabular}




\section{Article}

\section{Controlled Formation of the Two-Dimensional \\ TTBC J-Aggregates in an Aqueous Solution}

Burak Birkan, Demet Glen, and Serdar zelik

J. Phys. Chem. B, 2006, 110 (22), 10805-10813 • DOI: 10.1021/jp0573846

Downloaded from http://pubs.acs.org on January 16, 2009

\section{More About This Article}

Additional resources and features associated with this article are available within the HTML version:

- $\quad$ Supporting Information

- $\quad$ Links to the 3 articles that cite this article, as of the time of this article download

- $\quad$ Access to high resolution figures

- $\quad$ Links to articles and content related to this article

- $\quad$ Copyright permission to reproduce figures and/or text from this article

View the Full Text HTML 


\title{
Controlled Formation of the Two-Dimensional TTBC J-Aggregates in an Aqueous Solution
}

\author{
Burak Birkan, ${ }^{\dagger}$ Demet Güilen, $*, \hbar$ and Serdar Özçelik*,\$ \\ Faculty of Engineering and Natural Sciences, Sabancı University, Orhanl-34956 Tuzla, Istanbul, Turkey, \\ Physics Department, Middle East Technical University, 06531 Ankara, Turkey, and Chemistry Department, \\ Izmir Institute of Technology, Urla-35430 Izmir, Turkey
}

Received: December 19, 2005; In Final Form: March 17, 2006

\begin{abstract}
Strong experimental and theoretical evidence was provided on the controlled formation of the two-dimensional $\mathrm{J}$-aggregates that were assembled in the herringbone morphology. The exciton-band structure formation of 1,1',3,3'-tetraethyl-5,5',6,6'-tetrachlorobenzimidazolocarbocyanine (TTBC) J-aggregates was investigated in ionic $(\mathrm{NaOH})$ aqueous solution at room temperature. The control was achieved by changing the [TTBC] at a given $[\mathrm{NaOH}]$, or vice versa, and was monitored through the changes in the absorption, fluorescence excitation, and emission spectra. Specific attention was paid to expose the excited-state structure and dynamics through simulations of the excitonic properties, which included diagonal energetic disorder and phononassisted exciton relaxation. Aggregates were characterized by an asymmetrically split Davydov pair, an H-band $\left(\sim 500 \mathrm{~nm}, 1300 \mathrm{~cm}^{-1}\right.$ wide, Lorentzian-like) and a J-band $\left(\sim 590 \mathrm{~nm}, 235 \mathrm{~cm}^{-1}\right.$ wide, with a band shape typical of a one-dimensional J-aggregate), whose relative intensities showed a strong dependence on the $[\mathrm{TTBC}] /[\mathrm{NaOH}]$. The H-band is favored by high $[\mathrm{TTBC}]$ or high $[\mathrm{NaOH}]$. An explanation of the control on the aggregate formation was given by correlating the changes in the absorption with the structural modifications and the subsequent changes in the dynamics, which were induced by variations in the dye and $\mathrm{NaOH}$ concentrations. The J-band shape/width was attributed to disorder and disorder-induced intraband phononassisted exciton relaxation. The intraband processes in both bands were estimated to occur in the same time scale (about a picosecond). It has been suggested that the wide energetic gap between the Davydov split bands $\left(3000 \mathrm{~cm}^{-1}\right)$ could get bridged by the excitonic states of the loosely coupled chains, in addition to the monomeric species at low [TTBC]. Phonon-assisted interband relaxation, through the band gap states and/or directly from the $\mathrm{H}$ - to the J-band, are suggested for accounting the difference between the bandwidths and shapes of the two bands. Energy transfer between the H-band and the monomeric species is suggested as crucial for tuning the relative strengths of the two bands.
\end{abstract}

\section{Introduction}

Cyanine dyes exhibit strong optical absorption and interesting excitonic properties upon self-assembling into mesoscopic J-aggregate morphologies. Importance and attraction of cyanine J-aggregates owes mostly to their successful utilization in photographic industry since their discovery by Jelley and Scheibe. ${ }^{1-3}$ They are often considered as model systems to mimic the properties of photosynthetic light-harvesting complexes. ${ }^{4}$ Recently, the use of J-aggregates of cyanines, especially TTBC, as an imaging probe in molecular cell biology has been shown. ${ }^{5,6}$ Furthermore, their interesting linear and nonlinear optical properties make them promising organic optical materials that could find applications such as ultrasensitive detection of biohazards to superradiance and ultrafast optical switching..$^{7-20}$

Cyanine dyes can be self-assembled into mesoscopic Jaggregates by short-range noncovalent interactions such as van der Waals interactions, $\pi-\pi$ interactions, and hydrogen bonding. Aggregation is a process that is driven mainly by dispersion forces among the nearest neighbor molecules created by the high polarizability of the $\pi$-electrons of the polymethine

* Corresponding authors. E-mail: serdarozcelik@iyte.edu.tr (S.Ö.); dgul@metu.edu.tr (D.G.). Telephone: +90-232 7507557 (S.Ö.); +90-312 2105060 (D.G.). Fax: +90-232 7507509 (S.Ö.); +90-312 2105099 (D.G.).

† Faculty of Engineering and Natural Sciences, Sabanc1 University.

+ Physics Department, Middle East Technical University.

$\S$ Chemistry Department, Izmir Institute of Technology. backbone of cyanine. ${ }^{21}$ Not only the chromophores and the variations in their side chain structures (like the folding of proteins), but also the additives such as salts, bases, and alcohols can strongly influence the mesoscopic morphologies of the cyanine aggregates. ${ }^{1-3,22-27}$ For example, hydrophobic groups attached to cyanines may influence the aggregate morphology due to the enhanced role of solvophobic forces. ${ }^{22-24}$ Recently, it was reconfirmed that addition of various salts and bases induced formation of J-aggregates of cyanine dyes. ${ }^{25}$ Kinetic studies indicated a direct transition from the monomer state to the J-aggregate state without involvement of intermediate states such as dimers and trimers. ${ }^{26}$ In contrast, application of supramolecular polymerization and hierarchical self-organization techniques resulted in controlled formation of $\mathrm{H}$-aggregates of a merocyanine dyes. ${ }^{27}$

Reaching a control on optical properties of molecular aggregates through manipulation of their mesoscopic morphologies is an important step toward the realization of devices with controllable excitonic properties. Despite a great number of studies on the spectroscopic properties, the relationship between molecular arrangements, dynamics, and mesoscopic excitonic properties are still deficient. To this end, we have investigated the absorption and emission properties of TTBC aggregates in the presence of sodium hydroxide. We have, furthermore, analyzed the optical properties through exciton calculations to gather an understanding of the correlation between the aggregate 


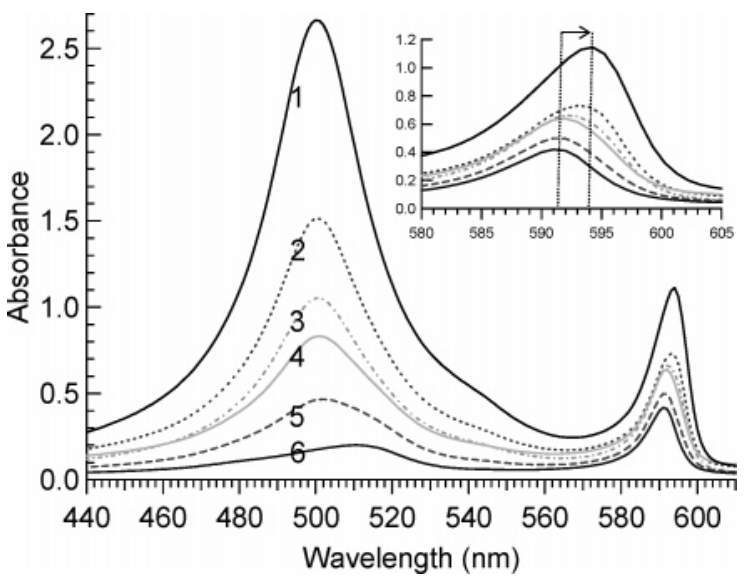

Figure 1. Set of absorption spectra of TTBC aggregates in the presence of $0.01 \mathrm{M} \mathrm{NaOH}$ solution. TTBC concentration varies in the solution, $0.9,0.5,0.4,0.3,0.2$, and $0.1 \mathrm{mM}$ numbered as 1 to 6 , respectively. Insert: expanded view of the spectra in the J-band region to clearly illustrate the red-shift of the peak position.

formation and the spectral changes that are imposed by the control of the dye/ion concentration. We provided convincing evidence on the controlled formation of two-dimensional aggregates in which two J-aggregate chains are assembled in herringbone morphology. A byproduct of the simulations has been a preliminary discussion of the dynamical processes.

\section{Experimental Methods}

Aggregation through its manifestation of exciton band formation can be employed to elucidate as to whether TTBC concentration can control the aggregate structure in aqueous solution of sodium hydroxide. A variety of mixtures of TTBC and aqueous solution of sodium hydroxide were prepared. Absorption and fluorescence spectroscopies were employed to investigate the excitonic properties of TTBC aggregates formed under the control of TTBC concentration at different sodium hydroxide concentrations.

1,1',3,3'-Tetraethyl-5, 5',6,6'-tetrachlorobenzimidazolocarbocyanine iodide salt (TTBC) was purchased from Hayashibara Biochemical, Co., Okayama, Japan, and used as-received. A stock solution of TTBC dissolved in methanol (spectroscopic grade) was prepared. Aggregates were prepared as follows: 1 $\mathrm{mL}$ of precisely known concentrations of TTBC, ranging from 0.1 to $1.0 \mathrm{mM}$, was mixed with $4 \mathrm{~mL}$ of aqueous solution of sodium hydroxide at a given concentration, varying from 0.01 to $1.00 \mathrm{M}$. The immediate color change of the dye solution from orange to bright pink indicates the formation of aggregates.

Absorption and fluorescence spectra were recorded, respectively, by a Varian Cary-5 UV-Vis-NIR and Cary Eclipse spectrophotometers, which have spectral resolutions better than 0.25 and $2.0 \mathrm{~nm}$. Fluorescence spectra were collected in the front-face geometry to eliminate reabsorption effects.

\section{Experimental Results}

Absorption Spectroscopy. Formation and structural variation of aggregates can be precisely monitored by UV-Vis absorption spectroscopy. Figure 1 shows a set of UV-Vis absorption spectra of the aggregates for various TTBC concentrations ranging from 0.1 to $0.9 \mathrm{mM}$ in $10 \mathrm{mM}$ aqueous solution of sodium hydroxide. A narrow and asymmetrical band (J-band) at $591 \mathrm{~nm}$ appeared at the lowest TTBC concentration. The J-band was red-shifted with respect to the band at $514 \mathrm{~nm}$, which resembles the monomer band (M-band) of TTBC in methanol.

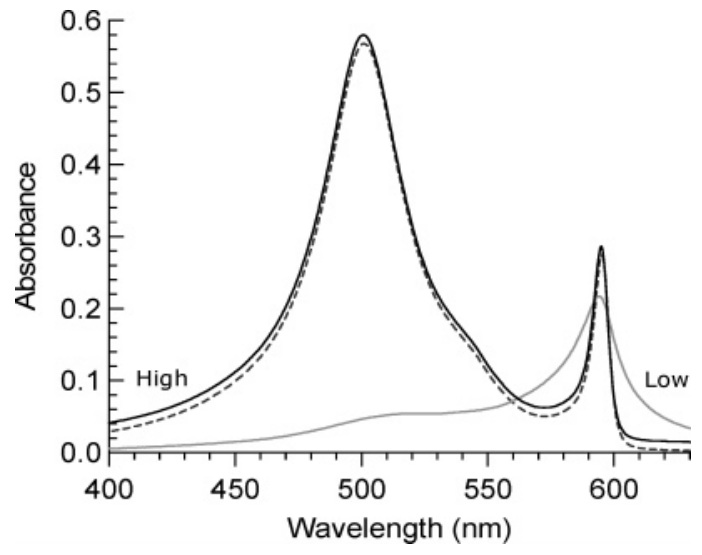

Figure 2. Absorption spectra of TTBC aggregates showing the influence of sodium hydroxide on the aggregate formation. The labels indicate the concentration of sodium hydroxide in the solution. $\mathrm{NaOH}$ concentrations are 0.01 (low), 0.10-1.00 M (high), while keeping TTBC concentration constant at $0.1 \mathrm{mM}$ in the solution.

As a result of progressive increase in TTBC concentration, a highly symmetrical and broad band (H-band) at $500 \mathrm{~nm}$, along with the J-band, appeared in the spectra, indicating the formation of another exciton state. The absorbance values of both J- and $\mathrm{H}$-bands became larger as the dye concentrations were progressively increased. The increase in the absorbance values can be attributed to a higher concentration of aggregates and/or to the increased size of aggregates. It is, however, experimentally very challenging to decipher and quantify the relative contributions of these factors to the absorbance. The ratio of absorbance of $\mathrm{H}$-band to J-band was increased linearly from 0.2 to $0.5 \mathrm{mM}$ concentrations of TTBC, then reached to saturation at the higher concentrations tested. The absorbance ratio of the peaks attained a steady value around 2.25. An absorption shoulder around 545 $\mathrm{nm}$ was an additional characteristic of the spectra. The Figure 1 insert illustrates the shift of the J-band peak to higher wavelengths accompanied with an increase in absorbance as the dye concentrations were increased. The incremental shift between the successive concentration steps is about $0.5 \mathrm{~nm}$. The total shift of the J-band peak is $3.0 \mathrm{~nm}$.

The shape of J-band is asymmetric, indicating an inhomogeneously broadened one-dimensional J-aggregate. ${ }^{28}$ The shape resembles a Gaussian on the red edge and is Lorentzian like on the blue edge. The J-band becomes more asymmetrical at higher dye concentrations. On the other hand, the H-band is extremely broad and exhibits a symmetrical Lorentzian-like shape at high TTBC concentrations (labeled as $1-3$ in Figure 1). Apart from the appearance of the monomeric shoulder at lower TTBC concentrations, the line shape and the peak position of the $\mathrm{H}$-band remains almost unaltered upon changes in the dye concentration.

To discern the role of sodium hydroxide on the spectral as well as excitonic properties, the absorption spectra of aggregates for a range of $\mathrm{NaOH}$ concentrations at a given TTBC concentration were obtained as shown in Figure 2. A single band at around $590 \mathrm{~nm}$ with a long tail was observed for the mixture of $0.05 \mathrm{mM}$ TTBC and $10 \mathrm{mM} \mathrm{NaOH}$. Aggregates exhibiting a dominant J-band formed favorably when the concentration of $\mathrm{NaOH}$ solution was low, typically in the range of mMs. The $\mathrm{H}$-band at $500 \mathrm{~nm}$ appeared in the presence of $100 \mathrm{mM}$ aqueous solution of sodium hydroxide without any diminishing of the J-band. In this condition, the exciton band was asymmetrically split into two bands, i.e., the H-band and the J-band.

The data presented above provide strong experimental evidence for the existence of at least two excitonic bands. The 


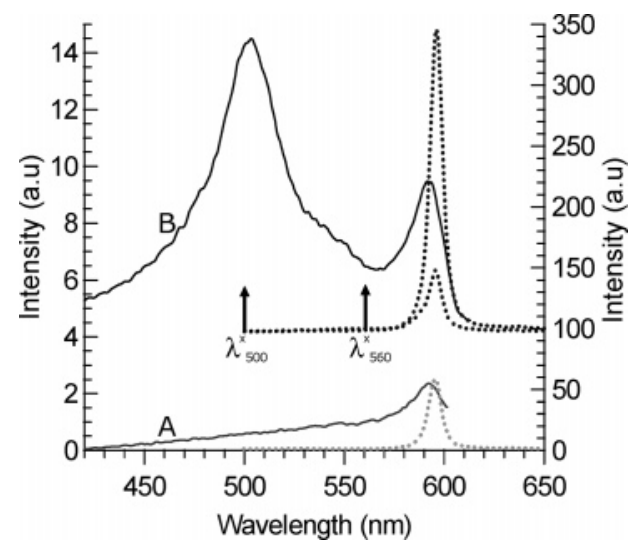

Figure 3. Fluorescence excitation (solid lines) and emission (dotted lines) spectra of TTBC aggregates. The scale of the excitation and emission spectra, rescaled, are on the left and the right axis, respectively. The baselines of the spectra are vertically shifted for the sample exhibiting the H-band, which has the following concentrations, [TTBC] $=0.05 \mathrm{mM},[\mathrm{NaOH}]=0.1 \mathrm{mM}$. The concentrations of the sample showing only the J-band are $[\mathrm{TTBC}]=0.05 \mathrm{mM},[\mathrm{NaOH}]=0.01 \mathrm{M}$. The excitations at 500 and $560 \mathrm{~nm}$ discriminate in emission intensity, leaving the band shape unaltered.

ability to tune the excitonic properties of aggregates with varying concentrations of TTBC and $\mathrm{NaOH}$ was, therefore, proven demonstrably. Because $\mathrm{NaOH}$ is a strong base, a weaker base, $\mathrm{N}$-methyl glucamine, was used in additional experiments. The same behavior of the formation of double bands and the progressive shift of the J-band peak was observed as with the $\mathrm{NaOH}$ used samples.

All the key features reported in this section is addressed further in the sections discussing the simulation study below.

Fluorescence Spectroscopy. The nature of the J- and $\mathrm{H}$-bands could be further explored by fluorescence spectroscopy as aggregates display emitting characteristics. Various samples were examined to identify the effect of concentrations on spectral properties. Figure 3 shows a representative fluorescence excitation and emission spectra of the samples. The spectra represented here is typical for the TTBC J-aggregates. The steady-state fuorescence spectrum appeared unaltered regardless of the concentrations of TTBC and $\mathrm{NaOH}$ and of the excitation wavelength. The fluorescence spectrum showed a very sharp and symmetrical band at $596 \mathrm{~nm}$ with full width at halfmaximum (fwhm) of $8.0 \mathrm{~nm}\left(185 \mathrm{~cm}^{-1}\right)$ at room temperature. An increase in fluorescence intensity was observed at higher TTBC concentrations. The excitation at $500 \mathrm{~nm}$ led to approximately a 5-fold increase in fluorescence intensity in comparison to that at $560 \mathrm{~nm}$. However, the fluorescence excitation spectra demonstrated two distinct features as a function of $\mathrm{NaOH}$ concentration. When the concentration was low, a single band (J-band) peaking around $591 \mathrm{~nm}$ along with a long tail on the blue side of the spectrum was obtained (A in Figure 3 ). This observation is consistent with the absorption spectra shown in Figure 1 displaying a single J-band (labeled as 6 in Figure 1). A shoulder at $545 \mathrm{~nm}$ appeared in the excitation spectrum (B in Figure 3 ) for the samples with high $[\mathrm{NaOH}]$, e.g., $0.10 \mathrm{M}$. The shape and the peak positions of the latter spectra are similar to those of absorption in Figure 1.

The appearance of the splitting in the absorption and the fluorescence excitation spectra associated with a "single" emission band in the fluorescence emission spectra could be taken as substantiation for the $\mathrm{J}$ - and the $\mathrm{H}$-bands belonging to the same excitonic manifold. A very broad and symmetrical shape of the H-band may be indicating an extremely fast exciton relaxation/energy transfer from the H-band. However, a very

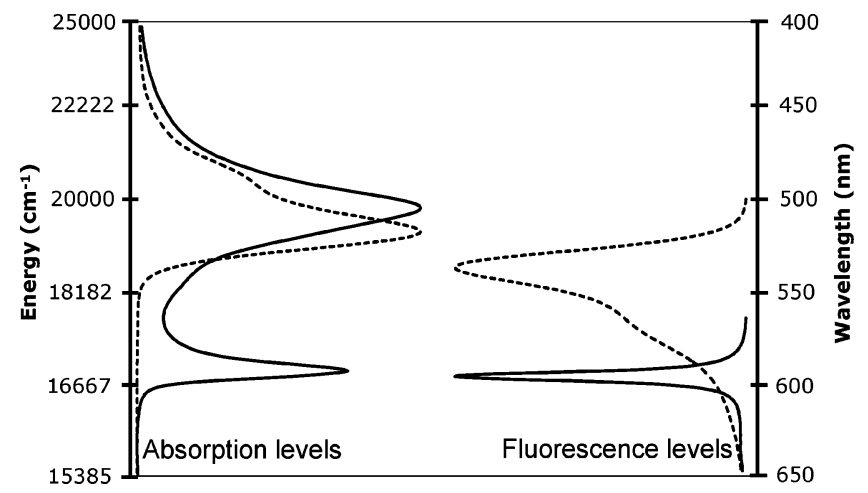

Figure 4. Energy levels of the TTBC aggregate and monomer (the dashed lines) to facilitate the possible exciton relaxation/energy transfer pathways. Normalized absorption and fluorescence spectra are separately illustrated for the sake of clarity.

fast energy transfer between the two different aggregates giving rise to the excitonic J- and H-bands cannot be entirely excluded.

To visualize the complex nature of the energetic structure of TTBC aggregates, the electronic spectra of the monomer and the aggregates were drawn in the same panel in Figure 4. Owing to the good overlaps between the absorption and emission of the monomer and aggregate bands, complex dynamical processes in the system can be expected.

We note that the Davydov splitting was postulated to be the case for 3,3'-bis(sulfopropyl)-5,5'-dichloro-9-ethylthiocarbocyanine (THIATS) aggregates as a result of several extensive experimental studies and modeling. ${ }^{29-33}$ The absorption spectra on the high-energy side of TTBC highly resemble the roomtemperature absorption spectrum of the THIATS aggregate in the water-ethylene glycol solution. ${ }^{31}$ In the THIATS aggregate, the $\mathrm{H}$ - and J-bands are almost symmetrically split around the monomeric transition, and the $\mathrm{H}$-band is more intense (about 35 times) than the J-band. The width and the shape of the $\mathrm{H}$-band are almost identical to the H-band observed in the spectra presented in this paper. Similarly, the J-band highly resembles the inhomogeneously broadened J-aggregate.

A recent study of the THIATS spectrum postulated that the $\mathrm{H}$ - and J-bands were Davydov split components resulting from the coupling of two aggregate chains in herringbone geometry. ${ }^{33}$ This geometry was named as two-strap. ${ }^{33}$ The intra- and interband exciton causing relaxations assisted by the molecular phonons were suggested as the mechanisms of the short lifetime of the H-band..$^{33}$

The numerous spectra that were presented in this paper offer an opportunity to verify this hypothesis, which was based on the interpretation of a single absorption spectrum.

\section{Model, Theory, and Numerical Procedures}

The absorption spectra were simulated on the basis of exciton calculations in the point-dipole approximation using the herringbone model. The band shapes/widths were attributed to diagonal energetic disorder (Gaussian inhomogeneous broadening) and exciton-phonon coupling (Lorentzian lifetime broadening). The inhomogeneous broadening was introduced using the method of Fidder et al. ${ }^{28}$ The lifetime broadening was treated in the limit of weak, linear, on-site exciton-phonon coupling. ${ }^{34-36}$

Herringbone Model. The herringbone model is illustrated in Figure 5. Each chain is a one-dimensional J-aggregate of $N_{\mathrm{J}}$ molecules. Each molecule is assumed to have a dipole-allowed transition between the ground state and the excited state of interest. This transition is specified by an excitation energy $E_{n}$ (equal to $E_{0}$ in the absence of disorder) and a transition dipole 

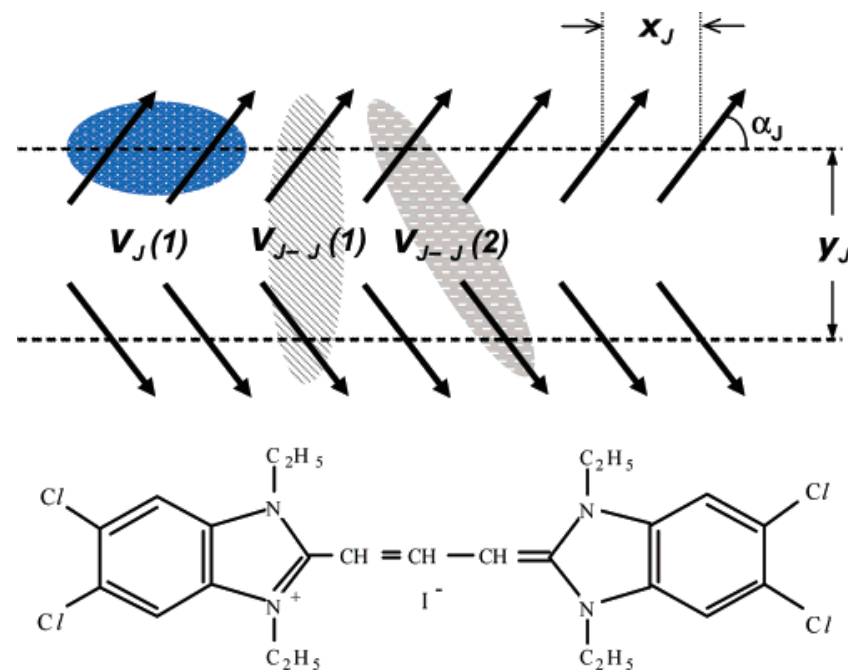

Figure 5. (a) Herringbone model. (b) Chemical structure of TTBC. The arrows represent the transition dipole moments. The transition dipole moment is assumed to be parallel to the long axis of the molecule. The leading interactions are indicated, but the simulations include all pairwise interactions.

moment $\overrightarrow{\mathbf{d}}_{n}=d_{n} \hat{\mathbf{d}}_{n}$ (we have assumed $d_{n}=d$ equal for all $n$ ). The dipoles are taken equidistant $\left(x_{\mathrm{J}}\right)$ and parallel to each other but at an arbitrary orientation relative to the line of aggregation $\left(\alpha_{\mathrm{J}}\right)$. The interchain separation is denoted by $y_{\mathrm{JJ}}$. The intrachain and interchain interactions were denoted, respectively, by $V_{\mathrm{J}}$ and $V_{\mathrm{J}-\mathrm{J}}$.

The independent morphological parameters of the model are $\alpha_{\mathrm{J}}$ and the factors $d^{2} / x_{\mathrm{J}}^{3}$ and $d^{2} / y_{\mathrm{J}-\mathrm{J}^{3}}$. The model with these three parameters offers flexibility of tuning the relative strengths of the Davydov split bands and provides the versatility of tuning the asymmetry in the band peaks. One may refer to Juzeliũnas and Knoester $^{37}$ for a quantitative analysis of the excitonic absorption spectrum within the herringbone model for homogeneous aggregates in the infinite chain length limit. Briefly, in this limit, the H-peak/J-peak absorbance ratio is $\operatorname{cotg}^{2} \alpha_{\mathrm{J}}$, and the peak maxima are located at

$$
\begin{aligned}
& E_{\mathrm{H}}(\max )=E_{0}+2 V_{\mathrm{J}}(1)+\left[V_{\mathrm{J}-\mathrm{J}}(1)+2 V_{\mathrm{J}-\mathrm{J}}(2)\right] \\
& E_{\mathrm{J}}(\max )=E_{0}+2 V_{\mathrm{J}}(1)-\left[V_{\mathrm{J}-\mathrm{J}}(1)+2 V_{\mathrm{J}-\mathrm{J}}(2)\right]
\end{aligned}
$$

where $V_{\mathrm{J}}(1), V_{\mathrm{J}-\mathrm{J}}(1)$, and $V_{\mathrm{J}-\mathrm{J}}(2)$ are defined in Figure 5 , and $E_{0}$ is defined above. Equations $1 \mathrm{a}-\mathrm{b}$ define the $\mathrm{H}$ - and $\mathrm{J}$-band edges that are on the red/at the bottom of both bands for $V_{\mathrm{J}}(1)$ $<0, V_{\mathrm{J}-\mathrm{J}}(1)>0$, and $V_{\mathrm{J}-\mathrm{J}}(2)<0$.

Theory. The Frenkel exciton Hamiltonian of an aggregate of $N$ molecules is ${ }^{38}$

$$
H_{\mathrm{ex}}=\sum_{n=1}^{N} E_{n}|n\rangle\left\langle n\left|+\sum_{n, m} V(n-m)\right| n\right\rangle\langle m|
$$

In eq $2,|n\rangle$ is the state in which the $n$th molecule of the aggregate is excited while all the others are in the ground state, $E_{n}$ is the excitation energy of the $n$th molecule (site energy), and $V(n-m)$ denotes the intermolecular interaction.

The diagonalization of $H_{\mathrm{ex}}$ gives the excitonic spectrum of the aggregate with the eigenstates $|v\rangle$ and the eigenenergies $E_{v}$, for $v=1, \ldots, N$,

$$
\begin{gathered}
|v\rangle=\sum_{n=1}^{N} C_{v n}|n\rangle \\
E_{\nu} C_{v n}=\sum_{m=1}^{N}\langle n|H| m\rangle C_{v m}
\end{gathered}
$$

In the case of dipolar interaction and in the point dipole approximation, $V(n-m)$ is given by

$$
V(n-m)=5.04 \frac{d_{n} d_{m} \kappa_{n m}}{r_{n m}{ }^{3}} \mathrm{~cm}^{-1}
$$

where $d_{n}$, the dipole moment strength, is in debye and $r_{n m}=$ $\overrightarrow{\mathbf{r}}_{n m} / \hat{\mathbf{r}}_{n m}$, the center-to-center distance from the $n$th molecule to the $m$ th molecule, is in nm. $\kappa_{n m}$ is given by

$$
\kappa_{n m}=\hat{\mathbf{d}}_{n} \cdot \hat{\mathbf{d}}_{m}-3\left(\hat{\mathbf{d}}_{n} \cdot \hat{\mathbf{r}}_{n m}\right)\left(\hat{\mathbf{d}}_{m} \cdot \hat{\mathbf{r}}_{n m}\right)
$$

The absorption strength of the $v$ th excitonic transition is

$$
D_{v}=\overrightarrow{\mathbf{d}}_{v} \cdot \overrightarrow{\mathbf{d}}_{v}
$$

with

$$
\overrightarrow{\mathbf{d}}_{v}=\sum_{n=1}^{N} C_{v n} \overrightarrow{\mathbf{d}}_{n}
$$

In eq 6, the absorption strengths are obtained for a particular realization of the site energies. The inhomogeneously broadened absorption spectrum is obtained by taking an average over an ensemble of a large number of aggregates, each of $N$ molecules and with a randomly generated set of uncorrelated site energies,

$$
D(E)=\left\langle\left(\sum_{v=1}^{N} D_{v} \delta\left(E-E_{v}\right)\right)\right\rangle
$$

where the function $\delta\left(E-E_{v}\right)$ is defined as $\delta\left(E-E_{v}\right)=1 / R$, for $\left|E-E_{v}\right| \leq R / 2$ and $\delta\left(E-E_{v}\right)=0$, otherwise with spectral resolution $R$.

The static disorder leads to (weak) localization of the exciton states. ${ }^{28}$ Inverse participation ratio for the states at energy $E$ is defined as 28

$$
\Lambda^{-1}(E)=\left\langle\sum_{\nu=1}^{N} \delta\left(E-E_{v}\right)\left(\sum_{n=1}^{N} C_{v n}{ }^{4}\right)\right\rangle /\left\langle\sum_{v=1}^{N} \delta\left(E-E_{v}\right)\right\rangle
$$

$1 / \Lambda^{-1}(E)$ is a good measure of the exciton localization length for well-localized states. ${ }^{28}$

The coupling of the weakly localized excitons to the molecular vibrations and/or to the vibrations of the host medium cause scattering between different exciton states. The generic expression for the transition rate from the exciton state $|v\rangle$ to the exciton state $|\mu\rangle$ in the limit of weak, linear, on-site excitonphonon coupling is: ${ }^{34-36}$

$$
\begin{aligned}
& W_{\mu \nu}=\sum_{n=1}^{N} S\left(\left|E_{v}-E_{\mu}\right|\right) \times \\
& C_{\mu n}{ }^{2} C_{v n}{ }^{2} x\left\{\begin{array}{ll}
\left\langle n_{\text {phonon }}\left(E_{\mu}-E_{v}\right)\right\rangle & E_{v}<E_{\mu} \\
1+\left\langle n_{\text {phonon }}\left(E_{v}-E_{\mu}\right)\right\rangle & E_{v}>E_{\mu}
\end{array}\right\}
\end{aligned}
$$

The transition rate is expressed in terms of the overlap integral of excitation probabilities for the states $|v\rangle$ and $|\mu\rangle$. The spectral function $S\left(\left|E_{v}-E_{\mu}\right|\right)$ contains the dependencies of the exciton- 
phonon coupling strength and of the phonon density of states on energy. The upper (the lower) expression stands for absorption (emission) of a phonon on site $n .\left\langle n_{\text {phonon }}\left(E_{\text {phonon }}\right)\right\rangle$ is the mean occupation number of a phonon state with energy $E_{\text {phonon }}$ at temperature $T$ and is taken as the Bose distribution,

$$
\left\langle n_{\text {phonon }}\left(E_{\text {phonon }}\right)\right\rangle=\left[\exp \left(\beta E_{\text {phonon }}\right)-1\right]^{-1}
$$

with $\beta=\left(k_{\mathrm{B}} \mathrm{T}\right)^{-1}$ and $k_{\mathrm{B}}$, the Boltzmann constant. The rates in eq 10 obey the principle of detailed balance, $W_{\mu \nu}=W_{\nu \mu}$ [exp$\left(\beta\left(E_{v}-E_{\mu}\right)\right]$, and the equilibrium (in the absence of radiative decay) is characterized by the Boltzmann distribution.

The width (fwhm) corresponding to the exciton state $|v\rangle$ is defined as

$$
\Gamma_{v}=\sum_{\mu \neq v} \hbar W_{\mu v}
$$

and for a Lorentzian absorption band of fwhm $\Gamma_{v}$, the time constant of relaxation is given by

$$
\tau_{v}=2 h / \Gamma_{v}
$$

Numerical Implementation. The Hamiltonian, $H_{\mathrm{ex}}$ (eq 2) is constructed in the point-dipole approximation by including all pairwise interactions in each $\mathrm{J}$-aggregate chain $\left(V_{\mathrm{J}}\right)$ and between the two chains $\left(V_{\mathrm{J}-\mathrm{J}}\right)$. The angular variable $\alpha_{\mathrm{J}}$ and the factors $d^{2} / x_{\mathrm{J}}^{3}$ and $d^{2} / y_{\mathrm{J}-\mathrm{J}}{ }^{3}$, which scale the intra- and interchain interactions, are treated as fit parameters. The eigenfunctions (eq $3 a$ ) and the eigenenergies (eq 3b) are obtained by numerically diagonalizing $H_{\mathrm{ex}}$ for a particular realization of site energies on the diagonal. The site energies are assumed to be uncorrelated random variables, each with a Gaussian distribution function centered at the monomeric energy $(514 \mathrm{~nm})$ and of the same fwhm $(\sigma) . \sigma$ is an empirical fit parameter. The inhomogeneous broadening is implemented by assigning the site energies out of these distributions at each cycle of random number generation, and a total of $10^{3}$ cycles are considered. Inhomogeneously broadened quantities (e.g., inverse participation ratio, eq 9) are obtained upon averaging over $10^{3}$ realizations of the disorder with $R=1.0 \mathrm{~cm}^{-1}$.

During each realization of disorder, the transition rates (eq 10) are calculated at room temperature. The spectral function $S\left(\left|E_{v}-E_{\mu}\right|\right)$, which scales the transition rate between the exciton states $|v\rangle$ and $|\mu\rangle$, is treated as an empirical fit parameter. Two different spectral functions are considered; one for the transitions from the H-band to the J-band, $S_{\text {inter }}(E)$, and one for the transitions within each band, $S_{\text {intra }}(E)$. Moreover, $S_{\text {intra }}(E)$ and $S_{\text {inter }}(E)$ are assumed to be constant for all transitions in the respective spectral regimes (see Simulation Results and Discussion). The widths calculated by eq 12 are used to execute the Lorentzian broadening at each cycle, i.e., eq 8 is employed by replacing $\delta\left(E-E_{v}\right)$ by the Lorentzian distribution of width $\Gamma_{v}$. The spectral digitization for the Lorentzian dressing is taken as $1.0 \mathrm{~cm}^{-1}$.

\section{Simulation Results and Discussion}

For the sake of simplicity, the simulations were confined to the long chain length limit. The absorption characteristics in the herringbone model, as in the case of one-dimensional J-/ $\mathrm{H}$-aggregates, have considerable size dependence only for very short chains $\left(N_{\mathrm{J}} \leq 15\right)$. For longer chains, the size-induced variations in the absorption spectra are minor compared to the changes induced by the remaining geometrical parameters $\left(\alpha_{\mathrm{J}}\right.$,
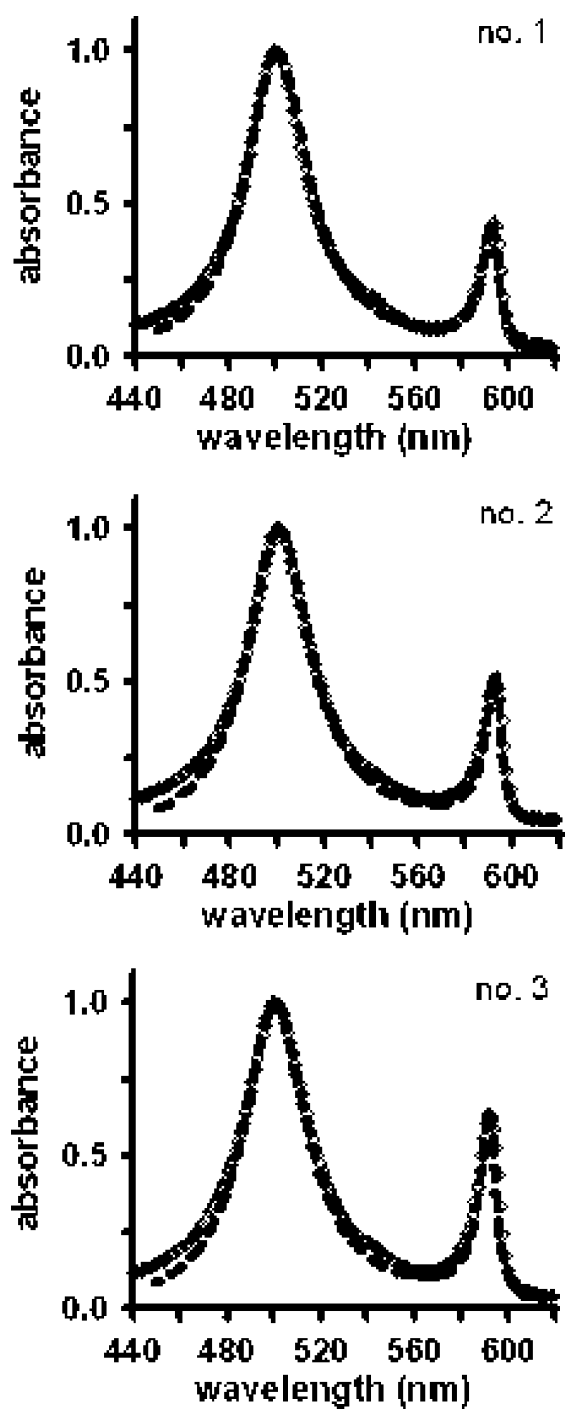

Figure 6. Simulations at a fixed chain length $\left(N_{J}=50\right)$ vs experimental absorption nos. 1-3. Experimental data (open diamonds) are normalized at the peak of the H-band. Simulations (dashed lines) are obtained upon averaging over $10^{3}$ cycles. The parameters common to all three simulations are, $\sigma=200 \mathrm{~cm}^{-1}, \hbar S_{\text {intra }}(E)=500 \mathrm{~cm}^{-1}$ and $\hbar S_{\text {inter }}(E)=$ $2500 \mathrm{~cm}^{-1}$. The geometrical parameters are given in Table 1 .

$y_{\mathrm{J}-\mathrm{J}}$, and $x_{\mathrm{J}}$ ). It suffices to say that the chain length mainly controls the absolute scale of the absorption spectrum.

Simulation of the High-Concentration TTBC Data. We first attempted a "simultaneous" simulation of the absorption spectra at high [TTBC] with no apparent monomeric contribution $(1,2$, and 3 in Figure 1). We use the term "simultaneous" to emphasize that the changes in the structure are kept systematic by allowing each geometrical parameter to change in a certain direction and by confining the search in a narrow interval. In addition, a fit with a common dynamics is aimed by keeping the set of band shape/width parameters, $S_{\text {intra }}(E), S_{\text {inter }}(E)$, and $\sigma$, fixed during the simulation.

In Figure 6 the simulations at a fixed size $\left(N_{\mathrm{J}}=50\right)$ are compared with the experimental data (nos. 1-3), where the experimental absorption spectra are normalized at the peak of the H-band. The morphological parameters of the herringbone model are $\alpha_{\mathrm{J}}, d^{2} /\left(x_{\mathrm{J}}\right)^{3}$, and $d^{2} /\left(y_{\mathrm{J}-\mathrm{J}}\right)^{3}$. In Table 1 , the parameters for the simulations in Figure 6 are listed as converted into distance information for two values of the monomeric absorption strength $(d)$. The values picked up for $d$ were in the range reported in the literature, which were also obtained on the basis of simulations of the spectra of cyanine aggregates. ${ }^{24,33}$ The band 
TABLE 1: Geometrical Parameters and the Leading Interaction Strengths for the Simulations, and the Comparison of the Jand H-Band Peak Positions for Homogeneous Aggregates in the Infinite Chain Length Limit with the Experimental Values

\begin{tabular}{|c|c|c|c|c|c|c|c|}
\hline expt no./ $\alpha_{\mathrm{J}}$ & $\begin{array}{c}d=8 \mathrm{D}: x_{\mathrm{J}}(\mathrm{nm}), \\
y_{\mathrm{J}-\mathrm{J}}(\mathrm{nm})\end{array}$ & $d=12 \mathrm{D}: x_{\mathrm{J}}(\mathrm{nm})$ & $\begin{array}{l}V_{\mathrm{J}}(1) \\
\left(\mathrm{cm}^{-1}\right)\end{array}$ & $\begin{array}{l}V_{\mathrm{J}-\mathrm{J}}(1) \\
\left(\mathrm{cm}^{-1}\right)\end{array}$ & $\begin{array}{l}V_{\mathrm{J}-\mathrm{J}}(2) \\
\left(\mathrm{cm}^{-1}\right)\end{array}$ & $\underset{(\mathrm{nm})}{E_{\mathrm{H}}(\max ) / \operatorname{expt}}$ & $\underset{(\mathrm{nm})}{E_{\mathrm{J}}(\max ) / \mathrm{expt}}$ \\
\hline $1 / 16.5^{0}$ & $\begin{array}{l}1.1054 \\
0.5476\end{array}$ & $\begin{array}{l}1.4433 \\
0.7176\end{array}$ & -419 & 2122 & -228 & $493.1 / 500.0$ & $590.0 / 593.5$ \\
\hline $2 / 18.0^{0}$ & $\begin{array}{l}0.0991 \\
1.0991 \\
0.5511\end{array}$ & $\begin{array}{l}0.1403 \\
1.4403 \\
0.7222\end{array}$ & -416 & 2111 & -226 & $493.1 / 500.0$ & $589.5 / 593.0$ \\
\hline $3 / 20.2^{0}$ & $\begin{array}{l}1.0869 \\
0.5561\end{array}$ & $\begin{array}{l}1.4242 \\
0.7287\end{array}$ & -412 & 2097 & -223 & $493.1 / 500.0$ & $589.0 / 592.5$ \\
\hline $4 / 22.0^{0}$ & $\begin{array}{l}1.0762 \\
0.5610\end{array}$ & $\begin{array}{l}1.4102 \\
0.7351\end{array}$ & -409 & 2086 & -220 & $493.1 / 501.0$ & $588.5 / 592.0$ \\
\hline $5 / 27.0^{0}$ & $\begin{array}{l}1.0310 \\
0.5732\end{array}$ & $\begin{array}{l}1.3510 \\
0.7512\end{array}$ & -406 & 2062 & -213 & $493.1 / 501.0$ & $588.0 / 591.5$ \\
\hline
\end{tabular}

shape/width parameters of the simulations were optimized at $\sigma$ $=200 \mathrm{~cm}^{-1}, \hbar S_{\text {intra }}(E)=500 \mathrm{~cm}^{-1}$, and $\hbar S_{\text {inter }}(E)=2500 \mathrm{~cm}^{-1}$. The magnitudes of the interaction strengths for each simulation and the homogeneous infinite chain peak maxima found using these strengths in eqs $1 \mathrm{a}-\mathrm{b}$ are also listed in Table 1. Also shown in this table are the parameters for the simulation of the experimental data nos. 4 and 5 (to be discussed under simulation of the low concentration [TTBC] data).

The simulations nos. 1-3 reproduced all the salient features of the absorption spectra including the positions of the J-and $\mathrm{H}$-peaks, the shapes of the bands, and their relative integrated absorption intensities, while the simultaneity criteria imposed was met reasonably.

The comparison of the analytical results with the experimental ones validates that the morphology and the nearest neighbor interactions are the key factors in determining the spectral positions, while the disorder and the dynamical processes that dominate the band shapes and widths account for further refinement of the spectral positions.

Interestingly, for the parameters given in Table 1 the two bands merge into a single absorption band at around $545 \mathrm{~nm}$ as the two J-aggregate chains become loosely coupled or uncoupled (see Figure 7). The presence of an absorption shoulder in the experimental spectra exactly around $545 \mathrm{~nm}$ can be interpreted as a further support for the herringbone morphology of the aggregates. It should be noted that this absorption shoulder, along with all the other spectral features observed in the absorption spectra, was also apparent in the fluorescence excitation spectra. However, a separate emission band from the $545 \mathrm{~nm}$ band was not observed in the fluorescence emission spectra. The close resemblance of the fluorescence excitation and absorption, and the absence of separate emission from the band gap states, can indicate a fast relaxation pathway between the $\mathrm{H}$ - and J-bands via the band gap states.

The reduction in the relative absorbance of the J-band and the red-shift of the J-peak at higher [TTBC] can be directly associated with smaller angles along the aggregation direction, with decreasing interchain separation, and with increasing intrachain molecular separation, $x_{\mathrm{J}}$. In addition, the possibility of formation of longer aggregates at higher dye concentrations cannot be excluded.

The variation in $\alpha_{\mathrm{J}}$ was less than $5.0^{\circ}$ between data no. 1 and data no. 3 . Owing to its range of $16.5-20.2^{\circ}$, the control of $\alpha_{\mathrm{J}}$ over the relative strengths of the two bands was by far dominant over the other parameters, which can be understood by referring to the characteristics of the homogeneous aggregate spectrum in the infinite chain length limit. In addition, the simultaneity criterion imposed was reasonably met also for the distance parameters, even at large values of $d$ (e.g., $d=12 \mathrm{D}$ ). It was also possible to achieve simultaneous simulations with different sets of bandwidth/shape parameters in the immediate

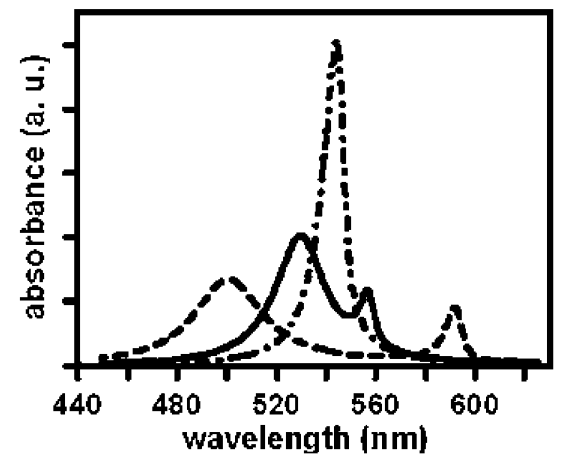

Figure 7. Merging of the Davydov split bands into a single excitonic band in the gap for the loosely coupled chains. The parameters common to all three simulations are, $N_{J}=50, d=12 \mathrm{D}, \alpha_{J}=20.2^{\circ}, x_{J}=$ $1.4242 \mathrm{~nm}, \sigma=200 \mathrm{~cm}^{-1}, \hbar S_{\text {intra }}(E)=500 \mathrm{~cm}^{-1}$, and $\hbar S_{\text {inter }}(E)=2500$ $\mathrm{cm}^{-1}, 10^{3}$ random cycles. The interchain distances, $y_{\mathrm{J}-\mathrm{J}}=0.73 \mathrm{~nm}$ (dashed), $y_{\mathrm{J}-\mathrm{J}}=1.00 \mathrm{~nm}$ (solid), and $y_{\mathrm{J}-\mathrm{J}}=3.00 \mathrm{~nm}$ (dashed-dotted).

neighborhood of the geometrical parameter sets given in Table 1 . For $\hbar S_{\text {inter }}(E)$ around $2500 \pm 50 \mathrm{~cm}^{-1}$, the values of $\sigma$ down to $175 \mathrm{~cm}^{-1}$ and up to $225 \mathrm{~cm}^{-1}$ with the respective $\hbar S_{\text {intra }}(E)$ of 520 and $480 \mathrm{~cm}^{-1}$ can yield similar simulation results.

The simulations and discussion presented above highly support that the features observed in the high [TTBC] spectra can be understood within a picture of J-aggregates coupled in the herringbone morphology. Moreover, a scheme of dynamics common to all data can be integrated by allowing rather minor changes in the geometry in this mesoscopic morphology.

Band Broadening versus Spectral Assignments and Localization Characteristics. Under the assumption that the coupling constants of the intraband scattering in the two bands are similar (which are represented by identical $S_{\text {intra }}(E)$ in the simulations), the energetic disorder and the intraband relaxation produce $\mathrm{J}$ and H-bands with comparable widths and shape (see Figure 8) or intraband relaxation occurs with about the same time scale in both bands. Therefore, the disorder-induced intraband broadening alone cannot account for the extremely large width and the symmetric shape of the H-band.

The disorder and the intraband relaxation for the most part account for the shape of the J-band. In addition, the red tail of the H-band, which extends all the way into the J-band, explains the absorption between 550 and $570 \mathrm{~nm}$ (see Figure 8b). An intraband relaxation time constant of 1.3 ps was estimated upon comparing the widths of the inhomogeneously broadened line shape $\left(200 \mathrm{~cm}^{-1}\right)$ and the lifetime broadened shape in the absence of $\mathrm{H}$-band broadening $\left(235 \mathrm{~cm}^{-1}\right)$. Around $85 \%$ of the absorption strength in the $\mathrm{H}$-band was contained in a very narrow spectral window (within $50 \mathrm{~cm}^{-1}$ of the H-band maximum) at the bottom of the $\mathrm{H}$-band. The average intraband relaxation time constant was estimated to be $1.2 \mathrm{ps} / 55 \mathrm{~cm}^{-1}$. 

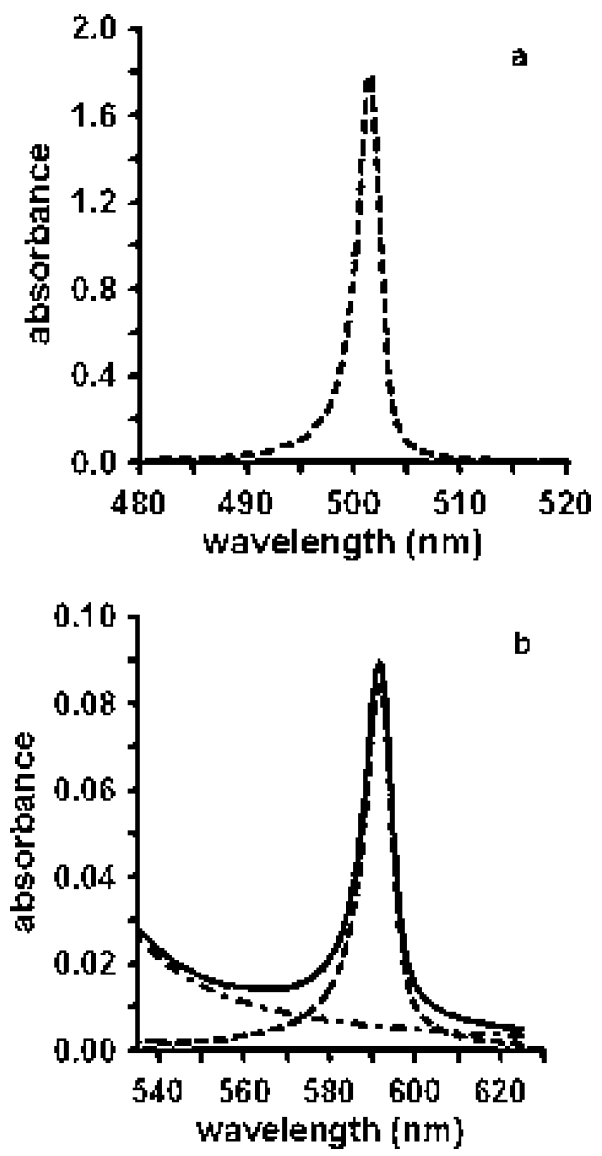

Figure 8. Band shape characteristics of the Davydov split bands. The simulation parameters are the same as in Figure 6, no. 3. Panel a: $\mathrm{H}$-band simulated with disorder and intraband relaxation. Panel b: J-band simulated with disorder and intraband relaxation (dashed), the tail of the H-band with $\hbar S_{\text {inter }}(E)=2500 \mathrm{~cm}^{-1}$ (dashed-dotted), and the sum of these two contributions (solid).

Therefore, a broadening of around $1200 \mathrm{~cm}^{-1} / 55 \mathrm{fs}$ remains to be attributed to the out-of-band relaxation of the H-band states.

It has not been our intention to describe the density of states and the localization characteristics of the exciton states in detail. It suffices to say that the weak localization of the excitonic states occurs upon inhomogeneous broadening. For instance, the inverse participation ratio (eq 9) evaluated for $N_{\mathrm{J}}=50$ with the disorder parameters mentioned above yielded a very similar extent of localization, around $0.3 N_{\mathrm{J}}$ sites, in the middle of the $\mathrm{J}$ - and H-bands. The band edge states were more localized, resulting about $0.1 N_{\mathrm{J}}$ sites on the red edge of the J-band, around $0.2 N_{\mathrm{J}}$ sites (on the blue edge of the J-band), and around 0.15 $N_{\mathrm{J}}$ sites on both edges of the H-band. This being the case, the difference in the overlap integrals/localization characteristics cannot account for an order of difference between the bandwidths.

Therefore, the width/shape of the H-band can only be explained by either using an intraband coupling constant of about an order of magnitude larger than that of the J-band (for which there seems to be no a priori reason), or it is necessary to suggest additional pathways that further shorten the lifetime of the H-band excitonic states.

In the interpretation of the THIATS spectrum by Basko et al., ${ }^{33}$ the molecular-phonon assisted interband relaxation was suggested to be responsible for the short lifetime of the H-band, with the added reservation that the phonon spectrum of such large organic molecules is rich enough to facilitate this relaxation across a band gap of about $3000 \mathrm{~cm}^{-1}$.

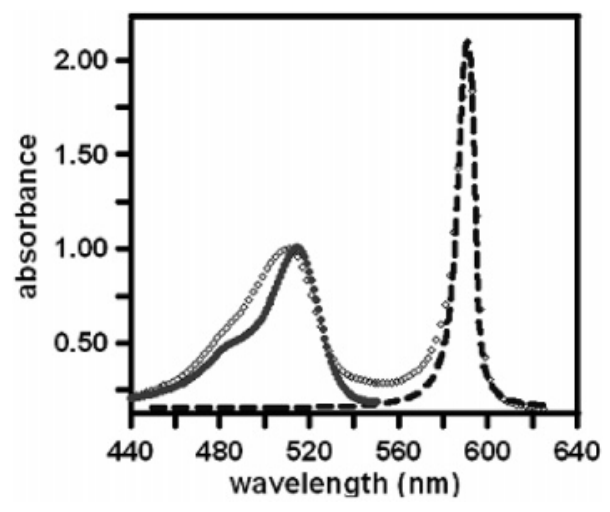

Figure 9. Experimental absorption (data no. 6 of Figure 1, open diamonds) vs monomeric absorption (closed diamonds) and a typical simulation for a one-dimensional chain (dashed lines). The experimental absorption is normalized at the peak of the $\mathrm{H}$-band. The simulation parameters are: $N_{\mathrm{J}}=50, \sigma=200 \mathrm{~cm}^{-1}, \hbar S_{\text {intra }}(E)=500 \mathrm{~cm}^{-1}$, and $\hbar S_{\text {inter }}(E)=2500 \mathrm{~cm}^{-1}, 10^{3}$ random cycles. The simulation of very loosely coupled chains follows at different $x_{\mathrm{J}}$ and $y_{\mathrm{J}-\mathrm{J}}$ for varying $\alpha_{\mathrm{J}}$. For example, for $d=8 \mathrm{D} ; \alpha_{\mathrm{J}}=15.0^{\circ}, x_{\mathrm{J}}=0.82 \mathrm{~nm}, y_{\mathrm{J}-\mathrm{J}}=2.00 \mathrm{~nm}$; $\alpha_{\mathrm{J}}=20.0^{\circ}, x_{\mathrm{J}}=0.77 \mathrm{~nm}, y_{\mathrm{J}-\mathrm{J}}=2.75 \mathrm{~nm} ; \alpha_{\mathrm{J}}=32.0^{\circ}, x_{\mathrm{J}}=0.71 \mathrm{~nm}$, $y_{\mathrm{J}-\mathrm{J}}=3.00 \mathrm{~nm}$.

In the absence of further experimental data, one cannot entirely disclaim this possibility. It is worthindicating the existence of an absorption shoulder in the band gap in the case of THIATS aggregates as well. ${ }^{29-33}$ As discussed above, in the presence of the band gap states $(545 \mathrm{~nm})$, the broadening of the $\mathrm{H}$-band can also be attributed to a phonon-assisted relaxation to the J-band that proceeds through the states in the gap while still not contradicting the fluorescence excitation and emission results. No simulations that explicitly take this pathway into account are provided in this paper. We believe that it should be possible to provide equally good simulations with a free fit parameter $S_{\text {inter }}(E)$. In either scattering scheme, interband scattering rates larger than intraband scattering rates are needed to explain the data.

Simulation of the Low-Concentration TTBC Data. The monomeric contribution, which is clearly visible in the data nos. 4 and 5, becomes more pronounced as the TTBC concentration decreases. At the lowest [TTBC], the band shape in the blue is very similar, but not exactly identical to that of the monomeric absorption in methanol (see Figure 9). It may be that the monomeric absorption is somewhat different in ionic environment, and the lowest [TTBC] spectrum is composed of a purely monomeric contribution and a J-band. Alternatively, a remaining trace of the Davydov split H-band may also be hidden under the wide monomer-like absorption band peaking around 514 nm.

A pure $\mathrm{J}$-aggregate band (or a band in which two J-aggregate bands with slightly different peak positions are merged) will naturally follow if the two chains become uncoupled (or loosely coupled). A typical simulation for very loosely coupled chains with the disorder and intraband parameters used in the high [TTBC] simulations is shown in Figure 9. It is clear that the superposition of these two components can give a reasonable reproduction of the experimental data no. 6. It should, however, be noted that, for a transformation of this type, rather extensive changes in the chain morphology are required. In the pure J-aggregate limit to obtain the right peak position (around 591 $\mathrm{nm})$, the interchain coupling of approximately $-1300 \mathrm{~cm}^{-1}$ is necessary in contrast to its value of around $-400 \mathrm{~cm}^{-1}$ in the presence of interchain coupling. A difference that large corresponds to reduction of $x_{\mathrm{J}}$ by about $30 \%$ at a fixed value of $\alpha_{\mathrm{J}}$. Such drastic structural changes seem quite unlikely in view of 


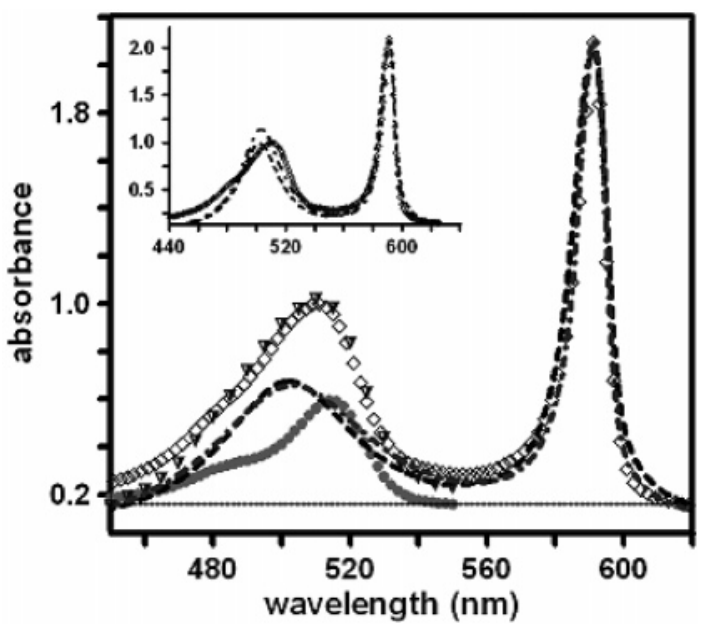

Figure 10. Simulation of the experimental data no. 6 within the herringbone model. Experimental data no. 6 normalized at the peak of the H-band (open diamonds), monomeric absorption in methanol (full circles). The parameters common to the simulations are: $d=8 \mathrm{D}, N_{\mathrm{J}}$ $=50, \sigma=200 \mathrm{~cm}^{-1}, \hbar S_{\text {intra }}(E)=500 \mathrm{~cm}^{-1}$, and $10^{3}$ random cycles. Dashed-dotted simulation: $\alpha_{J}=35.0^{\circ}, x_{\mathrm{J}}=0.91 \mathrm{~nm}, y_{\mathrm{J}-\mathrm{J}}=0.59$ $\mathrm{nm}$, and $\hbar S_{\text {inter }}(E)=4500 \mathrm{~cm}^{-1}$. Dashed simulation: $\alpha_{\mathrm{J}}=40.0^{\circ}, x_{\mathrm{J}}=$ $0.84 \mathrm{~nm}, y_{\mathrm{J}-\mathrm{J}}=0.61 \mathrm{~nm}$, and $\hbar S_{\text {inter }}(E)=3750 \mathrm{~cm}^{-1}$. The triangles correspond to the sum of the monomeric absorption band and the dashed simulation. The insert shows the corresponding simulations with $\hbar S_{\text {inter }^{-}}$ $(E)=2500 \mathrm{~cm}^{-1}$.

the smooth evolution observed in the remaining experimental data at various TTBC concentrations.

On the other hand, an H-peak with the band shape/width parameters discussed above in relation to the high [TTBC] simulations cannot be accommodated under the monomer-like band. Even at rather large $\alpha_{\mathrm{J}}$, at which the relative intensity of the H-peak is reduced, it is still necessary to further shorten its lifetime to mimic the monomer-like band shape observed at the lowest TTBC concentration. This discussion is communicated through the simulations given in Figure 10. An immediate possibility for "further" lifetime shortening is the energy transfer from the $\mathrm{H}$-band excitonic states to the monomeric species due to their energetic proximity.

The further broadening is not included in the simulations for nos. 4 and 5 shown in Figure 11. On the basis of the simulations/ discussion given above, it is clear that better reproductions would follow a superposition of the further-broadened $\mathrm{H}$-band and the monomeric spectrum. The inclusion of further broadening will force the geometry into a closer neighborhood of the simulations nos. $1-3$.

\section{Summary and Concluding Remarks}

The formation of TTBC aggregates in an ionic environment (aqueous solution of sodium hydroxide) was studied at room temperature. Absorption and fluorescence spectroscopies were employed to follow the dependencies of the aggregate/the excitonic band formation on the dye and ion concentrations. Experimental results that clearly point out that TTBC and sodium hydroxide concentrations, acting in concert, play a remarkable role in tuning the optical properties of TTBC aggregates have been presented.

Simulations of the absorption spectra at various TTBC concentrations at a given sodium hydroxide concentration were provided to expose the spectroscopic nature theoretically. Strong theoretical evidence was produced on the controlled formation of the two-dimensional aggregates in which two linear Jaggregate chains are assembled in the herringbone morphology.
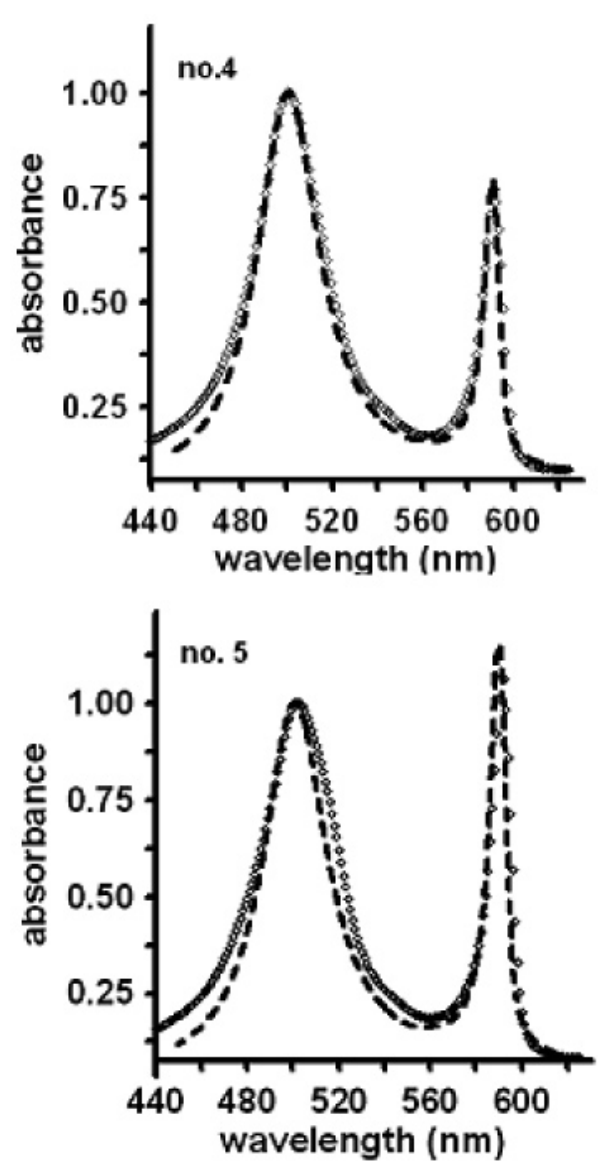

Figure 11. Simulations (dashed) vs experimental absorption, data no. 4 and 5 of Figure 1 (open diamonds). The experimental data is normalized at the peak of the H-band. Simulations are obtained upon averaging over $10^{3}$ cycles for $N_{\mathrm{J}}=50$ with the dynamical parameters $\sigma=200 \mathrm{~cm}^{-1}, \hbar S_{\text {intra }}(E)=500 \mathrm{~cm}^{-1}$, and $\hbar S_{\text {inter }}(E)=2500 \mathrm{~cm}^{-1}$, and with the geometrical parameters given in Table 1.

The aggregates were characterized by a Davydov split pair of exciton bands, which are highly asymmetrical around the monomeric transition $(514 \mathrm{~nm})$ and well-separated energetically $\left(\sim 3000 \mathrm{~cm}^{-1}\right)$. The blue band (H-band) around $500 \mathrm{~nm}$ is extremely wide $\left(\sim 1300 \mathrm{~cm}^{-1}\right)$ and has a symmetric Lorentzianlike shape, and the red band (J-band) around $590 \mathrm{~nm}$ resembles a typical one-dimensional J-aggregate band (fwhm around 235 $\mathrm{cm}^{-1}$ ). A strong dependence of the $\mathrm{J}$ - to $\mathrm{H}$-band intensity ratio as a function of TTBC concentrations at a given concentration of sodium hydroxide and vice versa was observed. The H-band was favored by high TTBC or high $\mathrm{NaOH}$ concentration.

The shape of the J-band was attributed to the disorder and intraband phonon-assisted exciton relaxation. The intraband processes in both bands were suggested to occur in the picosecond time scale and the intraband relaxation alone could not account for the shape/width of the H-band.

It has been suggested that the absorption shoulder is in the band gap (around $545 \mathrm{~nm}$ ). Additionally, the band gap was observed to get bridged by the monomeric species at low TTBC samples. It has been proposed that the existence of these states in the gap can evoke new pathways for a succession of energy transfer and exciton scattering processes for relaxation of energy from the H-band.

A relaxation proceeding via the $545 \mathrm{~nm}$ band was suggested as an alternative for the $\mathrm{H}$-band broadening that is consistent with the absorption and emission data presented in this study. Whether the broadening is due to a direct phonon-assisted 
relaxation between the $\mathrm{H}$ - and $\mathrm{J}$-bands $\mathrm{s}^{33}$ and whether these two pathways coexist remain as open questions.

An additional energy transfer from the $\mathrm{H}$-band states to the monomeric species was suggested to occur for low TTBC concentration samples. In the presence of energy transfer and phonon-assisted relaxation, it became viable to impart a smooth structural transformation involving minor differences in the geometry of molecular packing as the spectra changes from the limit of a very dominant H-band (high [TTBC]/high $[\mathrm{OH}]$ ) to the one with an $\mathrm{H}$-band almost nonexistent compared to the J-band (low [TTBC]).

Acknowledgment. This study was supported by a TÜBITAK (The Scientific and Technical Research Council of Turkey), grant TBAG-1939. We are very grateful to Professors J. Knoester and V. A. Malyshev (University of Groningen, The Netherlands) for stimulating discussions. H. Yıldırım's technical assistance during the preliminary stages of this work is also acknowledged. We also thank Dr. Gürhan Kalay for critical reading of the manuscript.

\section{References and Notes}

(1) Jelly, E. E. Nature 1936, 138, 1009.

(2) Scheibe, G. Angew. Chem. 1937, 50, 51

(3) Herz, H. Photogr. Sci. Eng. 1974, 18, 323.

(4) Van Amerongen, H.; Van Grondelle, R.; Valkunas, L. Photosynthetic Excitons; World Scientific: Singapore, 2000.

(5) Smiley, S.; Reers, M.; Mottola-Hartshorn, C.; Lin, M.; Chen, A.; Smith, T. W.; Steele, G. D., Jr.; Chen, L. B. Proc. Natl. Acad. Sci. U.S.A. 1991, 88, 3671 .

(6) Haugland, R. P. In Handbook of Fluorescent Probes and Research Products, 9th ed.; Gregory, J. Ed.; Molecular Probes, Inc.: Eugene, OR, 2002; pp 871-873. 6895 .

(7) Fidder, H.; Terpstra, J.; Wiersma, D. A. J. Chem. Phys. 1991, 94,

(8) Fidder, H.; Knoester, J.; Wiersma, D. A. Chem. Phys. Lett. 1990, $71,529$.

(9) Spano, F. C.; Mukamel, S. J. Chem. Phys. 1989, 91, 7988.

(10) Potma, E. O.; Wiersma, D. A. J. Chem. Phys. 1998, 108, 4894.

(11) Ozcelik, S.; Akins, D. L. J. Phys. Chem. B 1999, 103, 8926.

(12) Ozcelik, S.; Ozcelik, I.; Akins, D. L. Appl. Phys. Lett. 1998, 73, 1946.

(13) Ozcelik, S.; Akins, D. L. Appl. Phys. Lett. 1997, 71, 3057. 3143 .

(14) Yaglioglu, G.; Dorsinville, R.; Ozcelik, S. J. Appl. Phys. 2003, 94,
(15) Sato, Y.; Furuki, M.; Tian, M.; Iwasa, I.; Pu, L. S.; Tatsuura, S. Appl. Phys. Lett. 2002, 80, 2254.

(16) Mal'tsev, E. I.; Lypenko, D. A.; Shapiro, B. I.; Brusentsave, M. A.; Milburn, G. H. W.; Wright, J.; Hendriksen, A.; Berendtaev, V. I.; Kotov, B. V.; Vannikov, A. V. Appl. Phys. Lett. 1999, 75, 1896.

(17) Lidzey, D. G.; Bradley, D. D. C.; Armitage, A.; Walker, S. M.; Skolnick, S. Science 2000, 288, 1620.

(18) Jones, R. M.; Lu, L.; Helgeson, R.; Bergstedt, T. S.; McBranch,

D. W.; Whitten, D. G. Proc. Natl. Acad. Sci. U.S.A. 2001, 98, 14769.

(19) Glaeske, H.; Malyshev, V. A.; Feller, K.-H. J. Chem. Phys. 2001, 114, 1966.

(20) Markov, R. V.; Plekhanov, A. I.; Shelkovnikov, V. V.; Knoester, J. Phys. Status Solidi 2000, 221, 529.

(21) Bach, G.; Dahne, S. Cyanine Dyes and Related Compounds. In Second Supplements to the 2nd Edition of Rodd's Chemistry of Carbon Compounds; Sainsbury, M., Ed.; Elsevier: Amsterdam, 1997; Vol. IV, p 383.

(22) von Berlepsch, H.; Kirstein, S.; Bottcher, C. Langmuir 2002, 18, 7699.

(23) von Berlepsch, H.; Kirstein, S.; Bottcher, C. J. Phys. Chem. B 2003, 107, 9646.

(24) Didraga, C.; Pugžlys, A.; Hania, R. P.; von Berlepsch, H.; Duppen, K.; Knoester, J. J. Phys. Chem. B 2004, 108, 14976.

(25) Struganova, I. A.; Lim, H.; Morgan, S. A. J. Phys. Chem. B 2002, 106,11047

(26) Struganova, I. A.; Hazell, M.; Gaitor, J.; McNally-Carr, D.; Zivanovic, S. J. Phys. Chem. A 2003, 107, 2650.

(27) Wurthner, F.; Yao, Y.; Beginn, U. Angew. Chem. 2003, 42, 3247. 7880 .

(29) Scheblykin, I. G.; Bataiev, M. M.; Van der Auweraer, M.; Vitukhnovsky, A. G. Chem. Phys. Lett. 2000, 316, 37.

(30) Scheblykin, I. G.; Sliusarenko, O. Y.; Lepnev, L. S.; Vitukhnovsky, A. G.; Van der Auweraer, M. J. Phys. Chem. B 2001, 105, 4646.

(31) Scheblykin, I. G.; Drobizhev, M. A.; Varnavsky, O. P.; Van der Auweraer, M.; Vitukhnovsky, A. G. Chem. Phys. Lett. 1996, 261, 181.

(32) Scheblykin, I. G.; Varnavsky, O. P.; Verbouwe, W.; De Backer, S.; Van der Auweraer, M.; Vitukhnovsky, A. G. Chem. Phys. Lett. 1998 , 282, 250.

(33) Basko, D. M.; Lobanov, A. N.; Pimenov, A. V.; Vitukhnovsky, A. G. Chem. Phys. Lett. 2003, 369, 192.

(34) Leegwater, J. A.; Durrant, J. A.; Klug, D. A. J. Phys. Chem. B 1997, 101, 7205 .

(35) Shimizu, M.; Suto, S.; Goto, T. J. Chem. Phys. 2001, 114, 2775.

(36) Bednarz, M.; Malyshev, V. A.; Knoester, J. J. Chem. Phys. 2004, $120,3827$.

(37) Juzeliũnas, G.; Knoester, J. J. Chem. Phys. 2000, 112, 2325.

(38) Davydov, A. S. Theory of Molecular Excitons; Plenum: New York, 